\title{
A Logarithmic Photoreceptor Incorporating Lateral Bipolar Transistors for Improved Matching and Dynamic Range
}

\author{
Matthew D. Rowley, Member, IEEE, John G. Harris, Member, IEEE, and Shao-Jen \\ Lim, Member, IEEE
}

\begin{abstract}
Offsets and limited dynamic range in the photo-conversion elements of silicon VLSI vision systems limit the overall system performance in lowcontrast environments. This paper will introduce a new continuous-time, logarithmic photoreceptor which exhibits an improvement in the signal-to-offset ratio at low and medium intensities of $31 \%$ and $137 \%$ respectively. In addition, the logarithmic receptor exhibits a larger dynamic range than previous continuous-time receptors. Measured performance characteristics will be shown which quantify these improvements. We further show both analytically and through measured results that the offsets decrease as current levels increase.
\end{abstract}

\section{INTRODUCTION}

The first processing step in a fully integrated realtime, analog VLSI vision processing systems is the conversion of light energy into an electrical signal which can take on the form of a voltage, a current, or a charge. These photo-conversion elements (photoreceptors or receptors) can be designed to process information in various ways ranging from continuous-time analog [1] to time-adaptive analog [2] to discrete-time sampled [3] realizations. Comparisons among various types of receptors can be found in [4] and [5]. Each conversion method has an associated set of trade-offs and each also has a set of applications to which it is best suited. This paper will discuss a new continuous-time, logarithmic photoreceptor which is based on Mead's original receptor [1] but achieves a better signal-to-offset ratio and a larger dynamic range

Mead's original receptor has been used in many applications [6], [7], several of which have reported that offsets limited the system performance in low contrast environments. For this reason more recent designs [8], [9] have used time-adaptive receptors or photodiode conversion circuits [10] which are believed to be less susceptible to device mismatched offsets. The timeadaptive receptors are clearly zero-offset devices when no signal is applied. However when motion induces a response from the system, offsets are introduced into the solution by the other components comprising the photoreceptor circuitry. As for photodiodes, they inherently possess better matching characteristics than phototransistors, but since they operate at much lower current levels than phototransistors they typically require addi- tional amplification stages to process the input signals This additional circuitry again introduces offsets into the solution. To the authorrs' knowledge, no quantitative offset measurements of time-adaptive or photodiode conversion circuits have been reported

At low to moderate intensity ranges, the currents produced by the phototransistors are small enough to allow the PMOS load transistors in Mead's receptor to operate in the subthreshold region resulting in a logarithmic current-to-voltage relationship which increases the dynamic range. This introduces a trade-off since mosfets operating in subthreshold exhibit poor matching characteristics due to the exponential voltage-to-current relationship [11]. Section Il will introduce a new photoreceptor and discuss the fopology differences versus Mead's original photoreceptor. Section III will present the measured results from both receptors. Finally, Section IV will present our conclusions.

\section{The Lateral Bipolar Photoreceptor}

Figure 1 shows the schematic representations for both Mead's Original Logarithmic Photoreceptor (OLP) and for the Lateral Bipolar Photoreceptor (LBP). The phototransistors used in both receptors are identical in topology but the load devices are different. In the original receptor, PMOS load devices were used which exhibit a logarithmic response only while operating in the subthreshold region. In the LBP, however, lateral bipolar transistors (LBT) formed from PMOS devices operated in their lateral bipolar mode [12] have been used as the load devices. These devices not only provide a logarithmic current-to-voltage relationship over a larger range of currents, thereby increasing the dynamic range, but the matching characteristics of the LBTs are better than those for mosfets [13].

Several implementation trade-offs are associated with the use of LBTs. The first is that a larger physical layout area is required. The second is that there is no buried layer under the transistor to collect vertical currents thereby creating two bipolar transistors; one operating laterally and the other vertically as can be seen in 


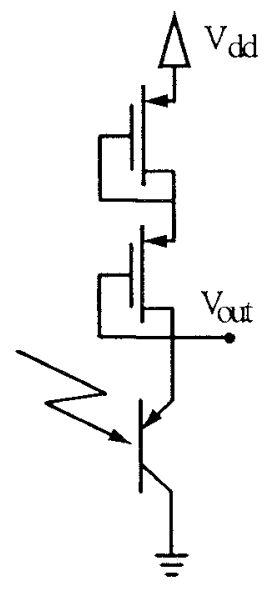

(a)

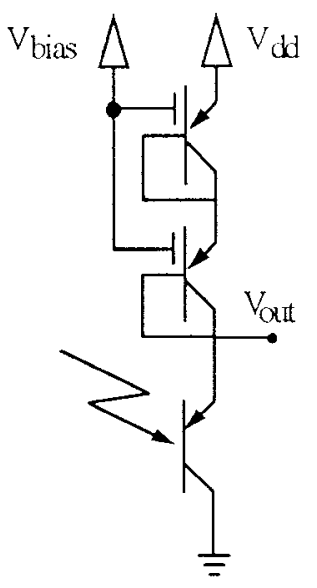

(b)
Figure 1. Schematic representations of Mead's 'Electronic Photoreceptor' in ' $a$ ' and of the Lateral Bipolar Photoreceptor in ' $b$ '. The phototransistors in both receptors are identical but the load devices have changed from PFETs operating in subthreshold to operating in their lateral bipolar mode thereby forming pnp transistors. The gate voltage of the LBTs can also be increased to improve matching characteristics [12].

Figure 2. The vertical bipolar effectively reduces the collector current from the lateral device which consequently reduces the effective transconductance. This could be remedied by using a technology incorporating buried collectors. Lastly, to achieve the best matching an additional bias supply is required for the gate teminal which exceeds the receptors emitter voltage. This bias is used to push the channel down into the substrate thereby moving the channel away from defects occurring along the silicon surface.

\section{MEASURED Results}

Both types of receptors were fabricated using the

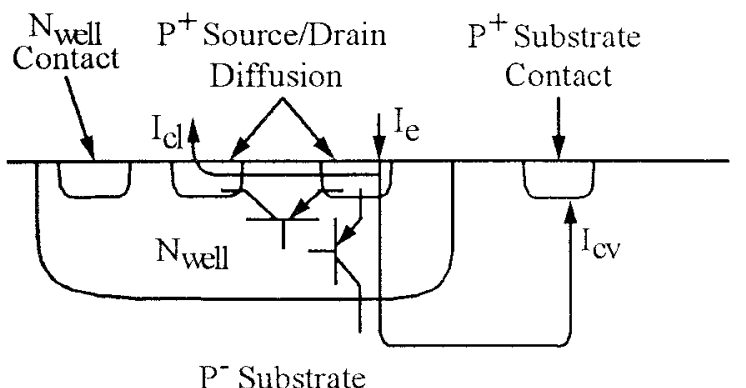

Figure 2. Schematic description of a lateral bipolar transistor showing the lateral and vertical transistors formed in a standard CMOS process without a buried collector.

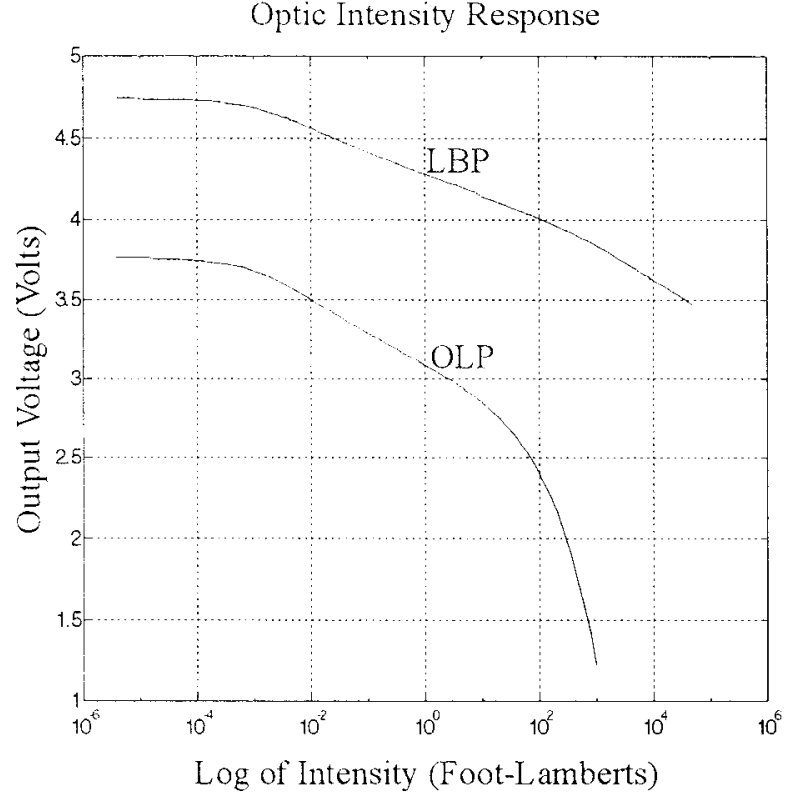

Figure 3. Photo-optic intensity response curves taken from the original photoreceptor and the lateral bipolar photoreceptor. The OLP response remained logarithmic over 4 to 5 orders of magnitude while the LBP remained logarithmic over 7 to 8 orders of magnitude illumination intensity.

$2 \mu \mathrm{m}$ Analog Nwell process from Orbit provided through MOSIS. Note that in the devices tested, the phototransistors used in the LBP were twice the size of the devices used in the OLP. Figure 3 shows the photooptic response from both receptors in units of FootLamberts. As can be seen, the original receptor remains logarithmic over 4 to 5 orders of magnitude of light intensity. The lateral bipolar receptor, however, remains logarithmic over 7 to 8 orders of magnitude. The LBP likely has an even larger dynamic range than the data suggests but the test equipment used to take these measurements could not exceed the optical intensities shown in the figure. Note also that the voltage levels produced by each receptor are different by almost a volt which must be accounted for in the design of subsequent processing circuitry.

Another difference between the two curves is the slope. The slope is a crucial parameter since it is a measure of the circuit's gain which thereby determines a systems resolution. The slope of the OLP is $207.5 \mathrm{mV}$ / decade while the slope for the LBP is $143.5 \mathrm{mV} /$ decade.

In generating the offset data for the photoreceptors, offsets were measured for 96 OLP devices and $108 \mathrm{LBP}$ devices respectively. Several different points along each curve were measured to examine the change in offsets with illumination level. Figure 4 shows the Gaussian distribution and nomalized histogram data for the offsets taken at two different illumination levels for the OLP devices. Note that the matching improves by $20 \%$ as the illumination spans the receptors dynamic range. 


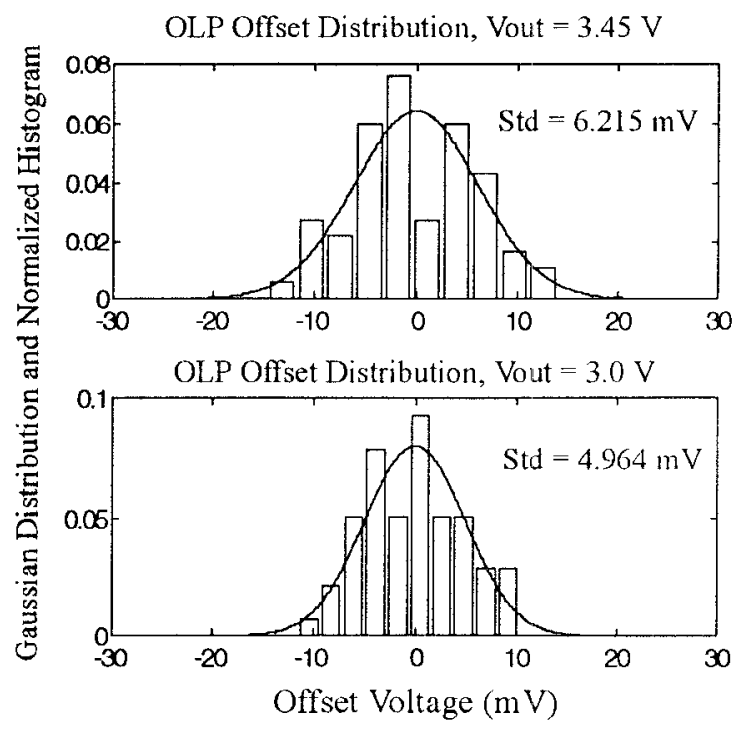

Figure 4. Gaussian distribution and normalized histogram of the offsets associated with the original logarithmic photoreceptor at two different illumination levels. Note that the matching improves as the current levels within the circuits increase.

The corresponding offset response for the LBP is shown in Figure 5. In this figure, the first two distributions correspond to the illumination levels shown in the

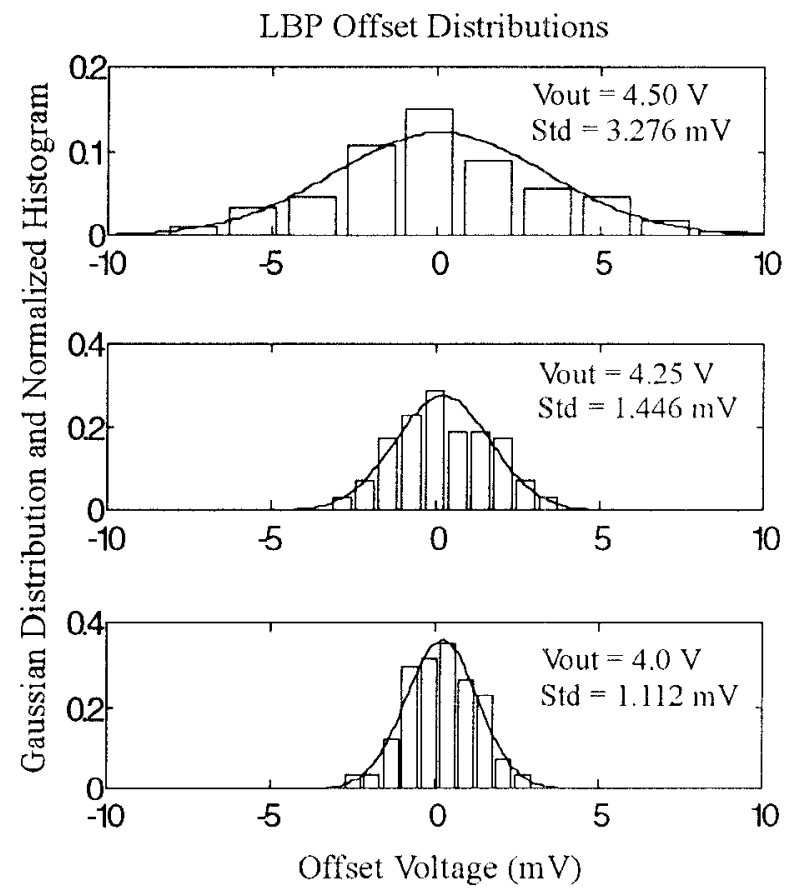

Figure 5. Offset distributions associated with the LBPs at three different illumination levels. The first two distributions correspond to the levels shown in Figure 4 for the OLPs. Again the matching improves as the current levels increase which lead to matching improvements of $47 \%$ and $70 \%$ respectively compared to the corresponding illumination levels in the OLPS. distributions of Figure 4 while the last was taken to determine matching levels at even higher illuminations. Again the matching improves as the current levels increase. Moreover, matching improvements of $47 \%$ and $70 \%$ respectively are observed when comparing the LBP versus the OLP for similar illuminations.

The corresponding signal-to-offset ratios are calculated by dividing the slope of the intensity responses by the standard deviation of the offsets. Therefore, the S/O ratios are 33.4 and 41.8 respectively for the OLP and $43.8,99.2$, and 129 respectively for the LBPs. The LBPs then exhibit an improvement in the S/O ratio of between $31 \%$ and $137 \%$ for similar intensities. Note, in the LBP realization tested here the LBT gate bias was not brought off-chip but was directly connected to Vdd. Therefore, matching characteristics can likely be further improved.

The decrease in offset clistributions with de current levels can be attributed to the transconductance-to-current ratio [11], [14]. Matching between transistors can be described as

$$
\frac{\Delta \mathrm{I}}{\mathrm{I}}=\Delta \mathrm{V}_{\mathrm{GS}} \cdot \frac{\mathrm{g}_{\mathrm{m}}}{\mathrm{I}}
$$

where $\Delta \mathrm{I}$ represents the current mismatch, $\mathrm{I}$ is the bias current, $g_{m}$ represents the transconductance, and $\Delta V_{G S}$ describes the change in gate voltage as a function of bias current. In the OLP, the equations describing the transconductance are [14], [15]

$$
g_{m}=\frac{I_{D}}{n U_{T}}
$$

in subthreshold where $U_{T}=k T / q$, and $n$ is the slope factor. In strong inversion, the transconductance is described by

$$
\mathrm{s}_{\mathrm{m}}=\sqrt{2 \beta \mathrm{I}_{\mathrm{D}}}
$$

where $\beta=\mu \mathrm{C}_{\mathrm{O}} \mathrm{W} / \mathrm{L}$. Also, in subthreshold

$$
\Delta \mathrm{V}_{\mathrm{GS}} \cong \mathrm{V}_{\mathrm{G}}-\mathrm{n} \mathrm{V}_{\mathrm{S}}=\mathrm{nU} \mathrm{U}_{\mathrm{T}} \ln \left(\frac{\mathrm{I}_{\mathrm{D}}}{\mathrm{I}_{\mathrm{S}}}\right)
$$

and in strong inversion,

$$
\Delta \mathrm{V}_{\mathrm{GS}}=\sqrt{2 \beta \mathrm{I}_{\mathrm{D}}}+\mathrm{V}_{\mathrm{TO}}
$$

In subthreshold one can see that $\mathrm{g}_{\mathrm{m}} / \mathrm{ID}_{\mathrm{D}}$ decreases slowly in proportion to $1 / \mathrm{n}$ while $\Delta \mathrm{V}_{\mathrm{GS}}$ increases proportional to the $\ln \left(\mathrm{I}_{\mathrm{D}}\right)$. Therefore the parameter of interest becomes $\Delta \mathrm{V}_{\mathrm{GS}} / \mathrm{I}_{\mathrm{D}}$ which decreases at a rate proportional to $\ln \left(I_{D}\right) / I_{D}$. Thus the ratio $\Delta I / I$ effectively decreases as the bias current increases. This effect continues into the strong inversion region where $\mathrm{g}_{\mathrm{m}} / \mathrm{I}$ decreases proportional to $\sqrt{\mathrm{I}_{\mathrm{D}}} / \mathrm{I}_{\mathrm{D}}$ while $\Delta V_{\mathrm{GS}}$ increases proportional to $\sqrt{I_{\mathrm{I}}}$.

A similar set of results can be shown for the LBP where

$$
\Delta V_{G S} \approx \Delta V_{B E}=U_{T} \ln \left(\frac{I_{C}}{I_{S}}\right)
$$


which closely resembles the subthreshold mosfet response shown in (4). In the LBTs, however, $g_{m} \sim I_{C}$ throughout its operating range therefore the parameter of interest is $\Delta V_{B E} / I_{C}$. As can be seen from (6), $\Delta \mathrm{V}_{\mathrm{BE}} / \mathrm{I}_{\mathrm{C}}$ decreases proportional to $\mathrm{ln}\left(\mathrm{I}_{\mathrm{C}}\right) / \mathrm{I}_{\mathrm{C}}$.

\section{CONCLUSIONS}

This paper introduces a new continuous-time logarithmic photoreceptor which exhibits a superior signalto-offset performance and a greater dynamic range than previous designs. The new photoreceptor matching characteristics improve as the dc bias current increases resulting in more than a 2 -to-1 improvement in the signal-to-offset ratio at moderate to high illumination levels. The new receptor is completely compatible with standard CMOS processes.

\section{ACKNOWLEDGMENTS}

The authors would like to thank the members of the Guided Weapons Evaluation Facility, Eglin AFB, FL. for providing technical support and expertise in testing these devices in addition to providing access to the necessary test equipment. Special thanks go to Dave Edwards, Jeff Estep, and Kurt Schroeder of the 46 TW! TSWGI.

\section{REFERENCES}

[1] C. Mead, "A Sensitive Electronic Photoreceptor," in 1985 Chapel Hill Conference on VLSI, H. Fuchs, Ed., Rockville: Computer Science Press, pp. 463-471, 1985.

[2] T. Delbruck, Investigation of Analog VLSI Visual Transduction and Motion Processing, Phd. Thesis, Computation and Neural Systems Program, Caltech, 1993.

[3] J. Wyatt et al., "Analog VLSI Systems for Image Acquisition and Fast Early Vision Processing," in International Journal of Computer Vision, 8:3, pp. 217-230, 1992.

[4] M. Sivilotti, M. Mahowald, and C. Mead, "Realtime Visual Computations Using Analog CMOS Processing Arrays," in Proc. Stanford Conf. on VLSI, MIT Press, Cambridge, MA., pp 295-312, 1987.

[5] R. R. Etienne-Cummings, Biologically Motivated Analog VLSI Systems for Optomotor Tasks, Phd. Thesis, University of Pennsylvania, 1994.

[6] M. A. Mahowald and C. Mead, "Silicon Retina," in Analog VLSI and Neural Systems, by C. Mead, Reading: Addison-Wesley, chp. 15, 1989.

[7] C. P. Chong, C. A. T. Salama, and K. C. Smith, "Image-Motion Detection Using Analog VLSI," in IEEE Journal of Solid-State Circuits, vol. 27, no. 1, pp. 93-96, Jan. 1992.
[8] M. A. Mahowald, "Silicon Retina with Adaptive Photoreceptors," in SPIE vol. 1473, Visual Information Processing: From Neurons to Chips, pp. $59-65,1991$

[9] T. Horiuchi, W. Bair, B. Bishofberger, A. Moore, and C. Koch, "Computing Motion using Analog VLSI Vision Chips: An Experimental Comparison Among Different Approaches," in International Journal of Computer Vision, 8:3, pp. 203$216,1992$.

[10] S. P. DeWeerth, "Analog VL.SI Circuits for Stimulus Localization and Centroid Computation," in International Journal of Computer Vision, vol. 8, pp. 19!-202, 1992.

[11] O. Schade, "BiMOS Micropower IC's," in IEEE Joumal of Solid-State Circuits, vol. SC-13, no. 6, pp. $791-798$, Dec. 1978

[12] E. A. Vittoz, "MOS Transistors Operated in the Lateral Bipolar Mode and Their Application in CMOS Technology," in IEEE Journal of SolidState Circuits, vol. SC-18, no. 3, pp. 273-279, Jun. 1983.

[13] T.W. Pan, and A. A. Abidi, "A 50-dB Variable Gain Amplifier Using Parasitic Bipolar Transistors in CMOS," in IEEE Journal of Solid-State Circuits, vol. 24, no. 4, pp. 951-961, Aug. 1989.

[14] E. A. Vittoz, "The Design of High-Performance Analog Circuits on Digital CMOS Chips," in IEEE Joumal of Solid-State Circuits, vol SC-20, no. 3, pp. 657-665, June 1985

[15] E. A. Vittoz et al. "MOS and Bipolar Transistors," in Practical Aspects of Analog and MixedMode IC Design: A 5-Day Intensive Course, vol. 1, Portland, OR, July 10-14, 1995. 